Métalepses du récit vidéoludique et reviviscence du sentiment de transgression

\title{
Sébastien Allain
}

\section{(2) OpenEdition}

\section{Journals}

Édition électronique

URL : http://journals.openedition.org/sdj/909

DOI : $10.4000 /$ sdj. 909

ISSN : 2269-2657

Éditeur

Laboratoire EXPERICE - Centre de Recherche Interuniversitaire Expérience Ressources Culturelles Education

\section{Référence électronique}

Sébastien Allain, « Métalepses du récit vidéoludique et reviviscence du sentiment de transgression », Sciences du jeu [En ligne], 9 | 2018, mis en ligne le 30 mai 2018, consulté le 01 mai 2019. URL : http:// journals.openedition.org/sdj/909 ; DOI : 10.4000/sdj.909

Ce document a été généré automatiquement le 1 mai 2019.

Tous droits réservés 


\title{
Métalepses du récit vidéoludique et reviviscence du sentiment de transgression
}

\author{
Sébastien Allain
}

1 La maturité d'un média se mesure en partie dans sa capacité à être réflexif, à se remettre en question et à briser les schémas canoniques qui l'ont d'abord institué. Littérature, théâtre, cinéma et bande dessinée ont tous vu l'éclosion de formes narratives dites «nouvelles », "modernes », «transgressives ». Parmi ces transgressions narratives, la figure conceptuelle de la métalepse a été observée dans chacun de ces domaines. Elle souligne la manière dont des niveaux narratifs normalement étanches se retrouvent alors reliés, allant jusqu'à l'interpénétration du monde raconté et du monde depuis lequel on raconte. En sa présence, l'expérience vécue s'apparente à un « jeu captivant » (Genette, 2004a) où la «suspension volontaire d'incrédulité» (Genette, 2004b, p. 23) - pacte de lecture classique pour la fiction - laisse place à une "simulation ludique de crédulité " (p. 25). Si la métalepse est un jeu en soi du point de vue de l'acte narratif, elle participa bien souvent aussi à renouveler l'interaction proposée aux lecteurs ou spectateurs. Mais là où la narration produisait une interaction symbolique, l'interaction au sens propre du jeu vidéo actualise désormais la narration. Avec le champ des récits interactifs, la métalepse devient donc omniprésente. Est-elle dès lors à reléguer dans les outils narratifs conventionnels? Est-elle devenue purement fonctionnelle? Une de ses formes ne pourrait-elle pas au contraire signer la modernité du jeu vidéo?

2 Pour répondre à ces questions, deux hypothèses sont avancées: (H1) la popularité de certaines transgressions narratives connues pour les précédents médias masque la diversité de celles spécifiquement proposées par le jeu vidéo ; et (H2) si la métalepse veut à nouveau offrir un sentiment de transgression, son omniprésence dans le jeu vidéo lui impose de renouer avec la narration. La méthodologie employée pour valider la première hypothèse consistera à confronter les définitions théoriques de la métalepse à une transgression très discutée pour le jeu vidéo notamment par des amateurs, journalistes, concepteurs de jeux vidéo : celle $\mathrm{du}$ « $4^{\mathrm{e}}$ mur ». Pour appréhender la seconde hypothèse, 
quelques exemples parmi la vingtaine de jeux de notre corpus seront plus particulièrement convoqués pour s'attacher aux possibles destinataires de l'effet de transgression: plutôt que viser directement le joueur, il s'agira d'utiliser l'interaction ludique pour viser le récit et les personnages eux-mêmes, avant d'envisager un réfléchissement vers le joueur. Par ce biais, quelques métalepses souligneront leur capacité à articuler ludique et narratif, et à revisiter leur nature transgressive, cette fois au service d'un récit vidéoludique.

\section{Le concept de métalepse}

\section{Aux origines}

Quel que soit le domaine dans lequel il était décrit, le principe de métalepse a provoqué un sentiment de transgression à quiconque l'observait. Qu'il s'agisse de rhétorique, linguistique, philosophie, ou d'arts comme la littérature, le théâtre ou le cinéma, la métalepse remet en question la logique instaurée, à toutes fins de surprendre, déranger, inquiéter parfois, amuser le plus souvent. Genette décrivit le principe au début des années 1970 dans le champ littéraire, avant de l'étendre au cinéma comme «toute intrusion du narrateur ou du narrataire extradiégétique [N-1] dans l'univers diégétique [N-2] (ou de personnages diégétiques [N-2] dans un univers métadiégétique [N-3], etc.) ou inversement » (Genette, 2007, p. 244) (voir Figure 1). Par ces intrusions, la métalepse remet ici en question la « frontière mouvante, mais sacrée entre deux mondes : celui où l'on raconte et celui que l'on raconte » (p. 245). Avec les années, la définition a été complétée pour considérer qu'il y a aussi métalepse lorsque "le passage d'un "monde" à l'autre se trouve, de quelque manière, masqué ou subverti " (Genette, 2004b, p. 117). Dès lors les mondes concernés peuvent tout aussi bien être du même niveau narratif ou concerner le niveau extratextuel ( $\mathrm{N}$ 0) sur lequel se situe le lecteur ${ }^{1}$.

Figure 1 : Niveaux narratifs basés sur l'ordonnancement de Genette (2007)

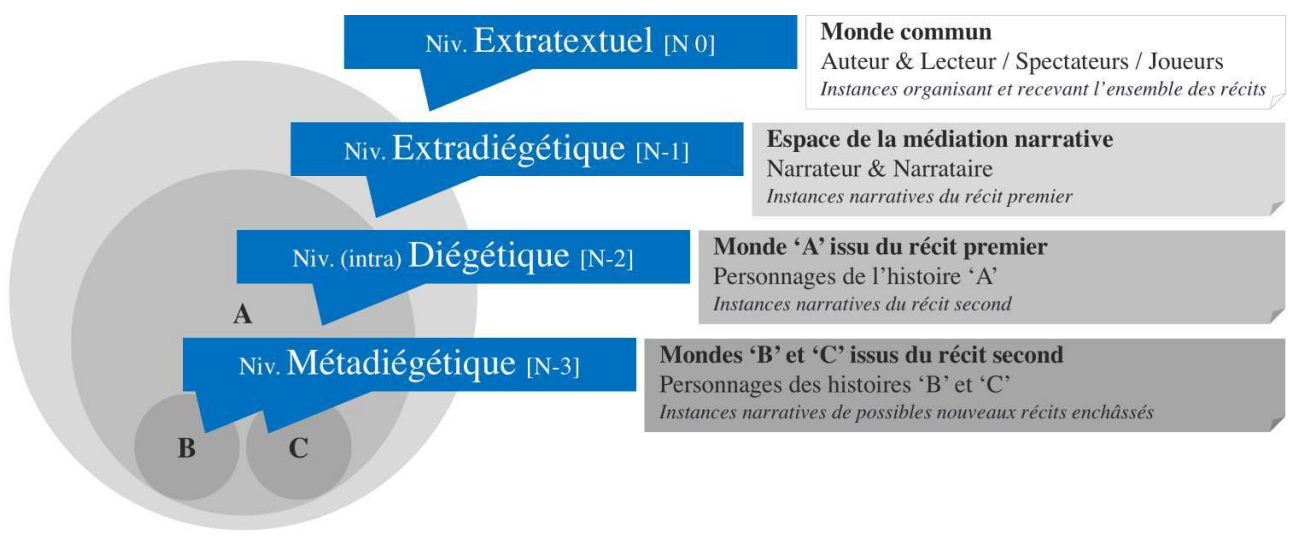

\section{Quelques critères distinctifs}

De nombreux critères ont progressivement vu le jour pour distinguer les métalepses entre elles, qualifiant notamment leur incidence dans le monde commun, leur localisation dans la temporalité du récit, leur fréquence et durée, leur orientation et direction, ainsi que leur type. Les trois derniers critères seront développés ici. 


\section{Orientation et direction}

5 L'orientation précise si le franchissement se fait horizontalement entre deux mondes d'un même niveau narratif (ex. B et $C$ de Figure 1) ou verticalement entre des niveaux narratifs enchâssés (ex. A et B). Dans ce cas, Cohn (2005) propose de distinguer les métalepses par leur position : les «métalepses extérieures » regroupent toutes celles qui se produisent « entre le niveau extradiégétique et le niveau diégétique, c'est-à-dire entre l'univers du narrateur et celui de son histoire» $(2005$, p. 122), alors que les «métalepses intérieures" se situent au sein d'une histoire et de récits enchâssés. Le terme « extérieures » peut porter à confusion au sens où il ne va pas jusqu'à intégrer le niveau extratextuel ( $\mathrm{N} 0)$. Il a par contre l'avantage de marquer l'espace de médiation $(\mathrm{N}-1)$ où siègent le narrateur et le narrataire (représentant symbolique du lecteur). Pour plus de précision, l'usage sera ici de qualifier la position en désignant le niveau d'origine de la transgression et celui de destination. Préciser l'origine et la destination a l'avantage de qualifier dans le même temps la direction, soit descendante ou ascendante, autrement appelée mouvement «intramétaleptique » ou "extramétaleptique » par W. Nelles (cité par Pier, 2005, p. 252), ou encore résumée par Genette comme le fait « d'entrer dans le cadre » ou de « sortir du cadre » (Genette, 2004b, p. 80).

\section{In verbis, In corpore}

6 Autre critère pour distinguer les métalepses, deux «types » principaux ont été stabilisés en narratologie (Ryan, 2004, 2005). Le premier regroupe les métalepses «rhétoriques » qui produisent un acte de communication ou de représentation sans déstabiliser les histoires. Par exemple, deux personnages du monde B pourront évoquer un personnage du monde $C$; quand bien même les protagonistes ne sont pas censés connaître l'existence d'un monde fictionnel parallèle à leur propre monde, les états de ces mondes ne seront pas affectés. Comme le dit Ryan, « l'opération n'a rien de contaminant, car elle respecte la différence des niveaux [ou espaces]. Elle ouvre une petite fenêtre sur un autre monde, mais elle la referme aussitôt » $(2005$, p. 207). Ce type regroupe également la notion de «pseudo métalepse » ou «quasi métalepse » où la transgression n'est que feinte, à la manière d'une illustration en trompe-l'œil (Ryan, 2004) ou du regard vers la caméra décrit par Mitry (cité par Kronström, 2001). Avec l'exemple du regard vers la caméra a priori lancé en direction du spectateur, celui-ci sera a posteriori récupéré par l'univers du film (diégétisé) par une explication rationnelle: le spectateur comprendra quelques instants après, par le montage ou la mise en scène, que le regard s'adressait en fait à un personnage hors-champ, bord-cadre (Vernet, 1988, p. 12). Il n'y a donc qu'un soupçon de transgression.

7 Le second type de métalepses ne s'arrête pas au clin d'œil ou à l'aparté in verbis vu précédemment. Les métalepses dites « ontologiques » créent cette fois des circulations in corpore de personnages entre les niveaux narratifs. En littérature par exemple, un romancier pourra alors commenter sa narration en étant lui-même au cœur de l'action. Il y a ici transgression ontologique, car il ne peut logiquement pas écrire et commenter l'histoire qu'il est en train de vivre, sans quoi il s'invente lui-même. Autre exemple au cinéma, avec La rose pourpre du Caire (Allen, 1985), où Cécilia spectatrice du monde A n'est pas censée pouvoir traverser un écran de cinéma pour rejoindre Tom Baxter, personnage d'un film constituant le monde $\mathrm{B}$, et réciproquement. Par ces interpénétrations et 
contaminations, ce second type de métalepse s'apparente souvent au genre fantastique et plus largement à tout récit voulant souligner une part d'irrationalité.

Tableau 1 : Synthèse des critères retenus pour la métalepse narrative

\begin{tabular}{|c|c|c|}
\hline Critère & Déclinaisons & Exemples \\
\hline \multirow[b]{2}{*}{ Type } & $\begin{array}{l}\text { Rhétorique (quasi/pseudo-) } \\
\text { / Rationnelle }\end{array}$ & $\begin{array}{l}\text { Deux personnages du monde } B \\
\text { évoquent un personnage du monde } C \text {. }\end{array}$ \\
\hline & $\begin{array}{l}\text { Ontologique } \\
\text { / Non rationnelle } \\
\text { (fantastique) }\end{array}$ & $\begin{array}{l}\text { Deux personnages du monde } \mathrm{B} \\
\text { font irruption dans le monde } \mathrm{C} \text {. }\end{array}$ \\
\hline \multirow{2}{*}{ Orientation } & Horizontale & Voir exemples pour « Types ». \\
\hline & Verticale & Voir exemples pour « Position». \\
\hline \multirow{2}{*}{$\begin{array}{l}\text { Positon } \\
\text { (si orientation } \\
\text { verticale) }\end{array}$} & Extérieure & $\begin{array}{l}\text { Le narrateur (N-1) fait irruption dans le } \\
\text { monde A. }\end{array}$ \\
\hline & Intérieure & $\begin{array}{l}\text { Un personnage du monde A fait irruption } \\
\text { dans le monde } \mathrm{B} \text {. }\end{array}$ \\
\hline \multirow{2}{*}{$\begin{array}{l}\text { Direction } \\
\text { (si orientation } \\
\text { verticale) }\end{array}$} & $\begin{array}{l}\text { Descendante } \\
\text { (mouvement } \\
\text { intramétaleptique) }\end{array}$ & Voir exemples pour « Position». \\
\hline & $\begin{array}{l}\text { Ascendante } \\
\text { (mouvement } \\
\text { extramétaleptique) }\end{array}$ & $\begin{array}{l}\text { Un personnage du monde B évoque ou } \\
\text { rejoint le monde A. }\end{array}$ \\
\hline
\end{tabular}

\section{Métalepse et récits interactifs}

Longtemps cantonnée aux médias dits « linéaires », avec des interactions symboliques ou imaginaires, la métalepse a trouvé de nouvelles formes dans les récits interactifs. Pour Di Crosta (2009), les interfaces offrent désormais au joueur la possibilité d'interagir avec l'histoire, de «faire irruption dans le contexte de production de la narration » (Di Crosta, 2009, p. 144). En troquant cycliquement sa position de « lecteur » (N 0), il peut endosser soit celle réservée jusque-là au « narrateur » $(\mathrm{N}-1)$, soit celle d'un personnage (niveau N-2 et inférieurs). En effet, quand le lecteur est représenté par un avatar, il peut descendre en personne "incarner" le rôle offert au sein du monde fictionnel. À ces formes de métalepses descendantes, initiées par le joueur, répond une forme ascendante suggérée par Ryan (2005) dans le contexte de l'intelligence artificielle : la boucle de rétroaction. Chaque changement d'état narratif remontant d'un système pour se traduire en données interprétables sur le niveau extradiégétique peut être considéré comme une métalepse ontologique ascendante (affichage de scores, retour de force, etc.). En conséquence, à chacune de ses interactions, le joueur choisit d'actualiser un récit tout autant qu'il produit une histoire. 


\section{Briser le $4^{\mathrm{e}}$ mur}

Si le concept de métalepse est encore assez peu utilisé dans le domaine du jeu vidéo stricto sensu, il est une expression qui surgit immanquablement dès lors que l'on s'intéresse à ses transgressions narratives : celle du « $4^{\mathrm{e}}$ mur ». Mais d'où vient cette expression ? Est-elle tout à fait adaptée? Et plus que tout, ne masque-t-elle pas derrière une bannière la diversité des propositions spécifiques au jeu vidéo?

\section{Du théâtre à la BD}

L'origine du " $4^{\mathrm{e}}$ mur » se situe au XIX siècle dans un théâtre qui cherchait le réalisme et la vraisemblance. Le principe consista pour les comédiens à faire comme si la scène n'était plus seulement bordée de murs à l'arrière et sur les côtés, mais également sur la partie qui s'offre au public. Coupés métaphoriquement du monde commun, les comédiens ne devaient en aucun cas s'adresser au public, sous peine de briser ce mur imaginaire et dans le même temps la suspension d'incrédulité accordée par le public. Le contrepied fut pris au début du XXe siècle avec Brecht qui considéra que le théâtre devait être ouvert sur les problématiques du monde et dans l'interpellation du public, pour le conduire vers un jugement critique et une prise de conscience. L'expression « $4^{\mathrm{e}}$ mur » fut reprise au cinéma où l'approche réaliste prohiba à nouveau tout regard vers ou à la caméra, qu'il s'agisse d'ailleurs de fictions ou de documentaires. Le $4^{\mathrm{e}}$ mur s'apparentait alors tout autant à la caméra qu'à l'écran du spectateur qu'il ne fallait ni désigner ni rappeler à l'esprit. Il fallut attendre la nouvelle vague pour voir les contre-exemples se multiplier, dont celui mémorable de Belmondo alias Michel Poiccard dans À bout de souffle (Godard, 1960), prenant à partie le public en se tournant ostensiblement vers la caméra, provoquant surprises et remises en question. Comme le souligne Beaulieu (2015), l'acte de briser le $4^{\mathrm{e}}$ mur est « brutal, souvent surprenant, rarement gratuit ». Sur les mêmes codes d'interpellation, avec un ton plus léger, la bande dessinée a elle véhiculé l'expression avec l'emblématique personnage de Deadpool depuis les années $90 .{ }^{2}$

\section{Un $4^{\mathrm{e}}$ mur au jeu vidéo?}

11 Et le domaine du jeu vidéo s'est lui aussi emparé de l'expression. Toutefois, l'on comprend déjà combien celle-ci a évolué d'un média au suivant. Au-delà du vocable (mur), il s'agit de vérifier si l'on parle toujours de la même transgression. Pour s'en convaincre, des exemples d'usage ont été ici sélectionnés et analysés. Dans une première phase, ceux-ci ont été recherchés via le moteur grand public Google, en croisant les expressions « $4^{\mathrm{e}}$ mur / $4^{\text {th }}$ wall » et «jeu vidéo / video game ». L'objectif était d'amasser des descriptions textuelles ou illustrations sous forme de vidéos ou d'images. La démarche ne prétendait pas à l'exhaustivité et visait les exemples déjà discutés et considérés comme acquis par la communauté. Six synthèses ont été retenues sur les dernières années pour la densité de leurs exemples, une cinquantaine à elles toutes, issus d'une quarantaine de jeux. Les auteurs sont des amateurs, journalistes, concepteurs et/ou enseignants en jeu vidéo (D’Argenio, 2016b ; GamingBolt, 2016 ; Griffiths, 2016 ; Owen, 2013 ; WatchMojo.com, 2014 ; Young, 2016). À ces synthèses se sont ajoutés deux articles, à mi-chemin entre billets de blogues et articles académiques, où les exemples cités recoupent ceux 
précédemment répertoriés, accompagnés d'une réflexion avancée sur le sujet (Conway, 2009 ; Turcev, 2014). Une réduction du corpus a ensuite été opérée avec comme critère l'intelligibilité. Ont été retenus les exemples qui bénéficiaient soit de descriptions croisées et concordantes entre auteurs, soit d'une explication ou illustration univoque. Le corpus ainsi restreint regroupa 22 exemples parmi 20 jeux. ${ }^{3}$

Restait à vérifier chaque fois s'il s'agissait bien de la fameuse transgression du $4^{\mathrm{e}}$ mur. Dans la continuité du court historique qui précède, l'expression "briser le $4^{\mathrm{e}}$ mur » s'apparente au sens strict à une métalepse rhétorique ascendante prenant son origine auprès d'un personnage diégétique (niveau $\mathrm{N}-2$ ou inférieur), et ayant pour destination un interlocuteur sur le niveau extratextuel ( $\mathrm{N} 0)$, en sa qualité précisément de lecteur, spectateur ou ici joueur. Suivant cette définition, ne restent alors plus que 7 exemples en lice, soit un tiers seulement du corpus restreint. À titre indicatif, l'analyse du corpus complet livre une proportion analogue. Quelques exemples et contre-exemples sont donnés ci-dessous, basés sur les descriptions des auteurs. ${ }^{4}$

\section{Les exemples}

De la BD au jeu vidéo, le personnage de Deadpool conserve sa caractéristique principale : s'adresser au lecteur. Avec sa version vidéoludique (High Moon Studios, 2013), il fait référence au fait d'être dans un jeu, se plaint du scénario et des dialogues, ou encore souligne au joueur la chance qu'il a de faire équipe avec Deadpool. Si chacune de ces interventions prises isolément constitue des exemples en soi, nous reviendrons plus loin sur le fait que le jeu dans son ensemble reposant sur ces transgressions ironiques, il fait finalement plutôt figure de contre-exemple. Autre exemple avéré, celui-ci ponctuel avec Metal Gear Solid (Konami, 1998) où le personnage de Psycho Mantis défie le joueur de poser sa manette, pour lui démontrer sa puissance télé kinésique en la faisant se déplacer (par vibration). Dernier exemple parmi les plus cités, Sonic the Hedgehog (Sonic Team, 1991), le personnage phare de l'enseigne SEGA, se montre impatient, regarde le joueur et tape du pied lorsque celui-ci n'interagit pas pendant plusieurs secondes sans avoir mis le jeu en pause. S'il n'y a pas de texte prononcé ici, l'exemple se range aussi dans le cas de métalepses rhétoriques brisant le $4^{\mathrm{e}}$ mur.

\section{Les contre-exemples}

Deadpool donne également l'occasion de pointer un contre-exemple lorsque le personnage-joueur rejoint soudainement le niveau de l'interface pour se saisir d'éléments comme les jauges de points et les utiliser comme armes contre ses adversaires. Si la métalepse est toujours ascendante, la transgression fusionne le niveau intra diégétique ( $\mathrm{N}-2)$ avec le niveau extradiégétique ( $\mathrm{N}-1)$, et non pas le monde commun ( $\mathrm{N}$ 0). Idem avec Monkey Island 2 (Lucasfilm Games, 1991) où le personnage-joueur a l'occasion de téléphoner au service support de Lucasfilm Games pour progresser dans l'énigme. La métalepse est alors ascendante et ontologique, avec un niveau d'origine métadiégétique (N-3), et un niveau de destination intra diégétique (N-2), sur lequel se trouve le développeur étant censé avoir donné vie au jeu. Un autre cas analogue est repérable dans la série des Pokémon (Game Freak, 1996) ${ }^{5}$ où le personnage pourra rencontrer toute l'équipe qui a conçu le jeu. Dernier contre-exemple, de nombreux éditeurs se sont ingéniés pour protéger leurs ventes en liant le jeu à un code. Dans le cas de Metal Gear Solid (1998) et StarTropics (Nintendo R\&D3, Locomotive Corporation, 1990), le 
joueur doit comprendre que le jeu lui glisse des indices au sein des dialogues entre personnages, pour qu'il se réfère à ce qui est inscrit sur la boite du jeu ou le fascicule original. Cet aspect allusif marque bien là aussi une différence avec l'interpellation ou l'aparté directement adressé correspondant au principe de briser le $4^{\mathrm{e}}$ mur. Pour ces deux jeux, si transgression d'une frontière il $\mathrm{y}$ a, elle est ontologique et descendante, faisant qu'un code passe du niveau extratextuel ( $\mathrm{N}$ 0) au niveau ( $\mathrm{N}-2)$, permettant de débloquer la suite de l'histoire. En somme, ces exemples communément rattachés au principe du $4^{\mathrm{e}}$ mur s'en départissent pour révéler une diversité de transgressions possibles.

Tableau 2 : Synthèse des exemples et contre-exemples cités

\begin{tabular}{|c|c|c|c|c|c|c|c|c|}
\hline \multicolumn{4}{|c|}{ Description du jeu } & \multicolumn{4}{|c|}{ Description de la métalepse } & \multirow{2}{*}{$\begin{array}{l}\mathbf{4}^{\mathrm{e}} \text { mur } \\
\text { Confirmation }\end{array}$} \\
\hline Nom du jeu & Date & Studio & $\begin{array}{l}\text { Moment } \\
\text { concerné }\end{array}$ & Position & Niveaux & Direction & Type & \\
\hline Deadpool & 2013 & $\begin{array}{l}\text { High Moon } \\
\text { Studios }\end{array}$ & $\begin{array}{l}\text { Prise à partie } \\
\text { du joueur par } \\
\text { Deadpool } \\
\text { (interventions } \\
\text { considérées } \\
\text { isolément) }\end{array}$ & Ext. & -20 & Asc. $\uparrow$ & Rhét. & Oui \\
\hline Deadpool & 2013 & $\begin{array}{l}\text { High Moon } \\
\text { Studios }\end{array}$ & $\begin{array}{l}\text { Deadpool se } \\
\text { saisit } \\
\text { d'élément de } \\
\text { l'interface pour } \\
\text { combattre }\end{array}$ & Ext. & $-2-1$ & Asc. $\uparrow$ & Onto. & Non \\
\hline $\begin{array}{l}\text { Metal Gear } \\
\text { Solid }\end{array}$ & 1998 & Konami & $\begin{array}{l}\text { L'interpellation } \\
\text { du joueur par } \\
\text { Psycho Mantis }\end{array}$ & Ext. & -20 & Asc. $\uparrow$ & Rhét. & Oui \\
\hline $\begin{array}{l}\text { Metal Gear } \\
\text { Solid }\end{array}$ & 1998 & Konami & $\begin{array}{l}\text { Saisie r d'un } \\
\text { code indiqué } \\
\text { sur la boite } \\
\text { pour débloquer } \\
\text { le récit }\end{array}$ & Ext. & -20 & Desc. & Onto. & Non \\
\hline $\begin{array}{l}\text { Monkey } \\
\text { island } 2\end{array}$ & 1991 & $\begin{array}{l}\text { Lucasfilm } \\
\text { Games }\end{array}$ & $\begin{array}{l}\text { L'appel } \\
\text { téléphonique } \\
\text { entre le } \\
\text { personnage et } \\
\text { la figure du } \\
\text { développeur }\end{array}$ & Int. & $-3-2$ & Asc. $\uparrow$ & Onto. & Non \\
\hline Pokémon & 1996 & Game Freak & $\begin{array}{l}\text { Rencontre du } \\
\text { personnage } \\
\text { avec l'équipe } \\
\text { du jeu qui l'a } \\
\text { créé }\end{array}$ & Int. & $-3-2$ & Asc. $\uparrow$ & Onto. & Non \\
\hline
\end{tabular}




\begin{tabular}{|c|c|c|c|c|c|c|c|c|}
\hline $\begin{array}{l}\text { Sonic the } \\
\text { Hedgehog }\end{array}$ & 1991 & Sonic Team & $\begin{array}{l}\text { L'impatience } \\
\text { de Sonic }\end{array}$ & Ext. & -20 & Asc. $\hat{\mid}$ & Rhét. & Oui \\
\hline $\begin{array}{l}\text { Stanley } \\
\text { Parable }\end{array}$ & 2013 & $\begin{array}{l}\text { Galactic } \\
\text { Cafe, } \\
\text { Everything } \\
\text { Unlimited } \\
\text { Ltd }\end{array}$ & $\begin{array}{l}\text { La contrariété } \\
\text { affichée } \\
\text { narrateur } \\
\text { envers } \\
\text { actions } \\
\text { joueur }\end{array}$ & Ext. & -10 & Asc. $\uparrow$ & Rhét. & Non \\
\hline StarTropics & 1990 & $\begin{array}{l}\text { Nintendo } \\
\text { R\&D3, } \\
\text { Locomotive } \\
\text { Corporation }\end{array}$ & $\begin{array}{lr}\text { Saisie } & \text { d'un } \\
\text { code à } & \text { révéler } \\
\text { par l'eau } & \text { pour } \\
\text { débloquer } & \text { le } \\
\text { récit } & \end{array}$ & Ext. & -20 & Desc. & Onto. & Non \\
\hline
\end{tabular}

\section{Un concept à bout de souffle} interactions possibles entre jeu et joueur. Toutefois, plutôt que de l'abandonner, il cherche à le rapprocher du "cercle magique " décrit par Huizinga ([1938] 1988), en proposant l'idée d'un "mur circulaire» capable de se contracter autour de la stricte diégèse ou de s'élargir jusqu'à englober joueur, matériel, manuels, boites et tout ce qui peut servir l'univers du jeu. Il ne parle dès lors plus de «briser» le $4^{\mathrm{e}}$ mur, mais de le « relocaliser » afin de faire varier l'immersion. La proposition de Conway peut se résumer dans le passage d'un $4^{\mathrm{e}}$ mur historiquement conçu comme une métalepse rhétorique venant d'un personnage à une métalepse ontologique permettant au monde commun $(\mathrm{N} 0)$ d'être annexé par la diégèse $(\mathrm{N}-2)$. Le problème à l'évidence est de brouiller le principe originel en voulant l'étendre à tout prix. Par ailleurs, comme le fait remarquer Turcev (2014), le "cercle magique" peut évidemment entourer le joueur sans même attendre d'évènements qui signifient l'annexion du monde commun, car «le joueur est par défaut inclus » dans ce cercle lorsqu'il joue. Et pour ces évènements ayant attrait au monde commun, le concept de "métalepses dispositives " proposé la même année par Bouchardon (2009) semble tout indiqué pour décrire l'extension de la fiction: les éléments extratextuels cités par la diégèse - comme un site internet pour résoudre une énigme, ou l'utilisation de SMS pour déclencher la suite du récit - étendent le « dispositif de lecture » et la fiction qui l'habite. ${ }^{6}$ C'est alors le contrat narratif qui s'en trouve enrichi, devenant "plus dense, parfois plus exigeant, à mesure que les ressources non diégétiques se multiplient » (Turcev, 2014). Nul besoin donc de se référer encore au $4^{\mathrm{e}}$ mur.

Orienté dans la seule direction ascendante et visant directement le joueur ( $\mathrm{N}$ 0), le « $4^{\mathrm{e}}$ mur » semble insuffisant pour théoriser le jeu vidéo. Par ailleurs, les exemples avérés de $4^{\mathrm{e}}$ mur transgressent-ils encore quelque chose ? Ne se contentent-ils pas trop souvent de copier ce qui a pu marcher un temps pour un autre média? À ces exemples narratifs peuvent s'ajouter des exemples esthétiques soulignant la présence de l'écran si ce n'est du «mur»: de nombreux effets de projections de salissure, de peinture ou de reflets de lumière sont répertoriés. Or, dès qu'ils ne sont pas justifiés par la présence de lunettes, 
d'un pare-brise ou d'une vitre dans la diégèse, ils soutiennent certes l'idée de la présence d'une caméra et de propriétés physiques réalistes qui la ferait être entachés (Conway, 2009), mais ils soutiennent surtout le simulacre d'une caméra qui n'existe plus en tant que telle pour ce média. ${ }^{7}$ Alors que l'inventivité des jeux vidéo tendrait à les distinguer des anciens médias et du cinéma dont il est un proche parent (Blanchet, 2008), le maintien de ce concept dans les exemples répertoriés traduit de fait une attente de la communauté. Les contre-exemples initialement regroupés sous le seul principe du $4^{\mathrm{e}}$ mur ont l'avantage de permettre de prendre conscience des transgressions sous-jacentes : en mettant en valeur différents niveaux narratifs, c'est autant d'autres formes qui se dévoilent déjà. La renommée du principe du $4^{e}$ mur permet de confirmer notre première hypothèse (H1) qui avançait que la popularité de certaines transgressions narratives connues pour les précédents médias masque la diversité de celles spécifiquement proposées par le jeu vidéo.

\section{Reviviscence du sentiment de transgression}

Si la métalepse s'avère un outil conceptuel extrêmement discriminant pour l'analyse, on peut encore s'interroger sur ce qu'il reste de son potentiel transgressif auprès du joueur, et le cas échéant de la manière de le renouveler. La question de son potentiel sera abordée d'un point de vue pragmatique et historique, avant de souligner quelques jeux vidéo exemplaires et d'y adjoindre les jeux dits sérieux. Enfin, la réponse concernant le potentiel de la métalepse s'attachera à ce que le joueur ne soit plus le destinataire premier de la transgression, comme avec le $4^{\mathrm{e}}$ mur, mais un destinataire second, par l'entremise d'un effet mimétique et empathique.

\section{Banalisation d'une figure?}

Le caractère transgressif de la métalepse est-il en voie d'extinction? Tout d'abord, sur le plan pragmatique et suivant les définitions données dans la partie Métalepse et récits interactifs, toute interaction du joueur, qu'elle intervienne sur l'histoire ou dans l'histoire lorsque celui-ci incarne un personnage, ou toute boucle rétroactive (feedback), relève d'une forme de métalepse. Dès lors, cette forme fonctionnelle est tellement omniprésente qu'elle est pour le moins banalisée. Paradoxalement, c'est désormais son absence qui produit une transgression du domaine vidéoludique: il suffira que le jeu retire momentanément le contrôle au joueur, l'empêchant de s'immiscer dans l'histoire ou de la voir s'actualiser, pour qu'apparaisse une rupture dans le contrat tacite de «lecture». L'exemple donné par Conway (2009) avec Sonic en est une illustration: Sonic impatient laissera le joueur démuni lorsqu'il finira par sauter dans le vide, le privant du contrôle et de la suite de l'histoire. En somme, si la métalepse a trouvé un nouveau terrain avec le jeu vidéo, le caractère transgressif des formes purement fonctionnelles s'y est rapidement épuisé.

Sur le plan historique, bon nombre d'auteurs indiquent pour la littérature que des apparitions répétées dans une œuvre seraient une autre source d'affaiblissement, finissant par l'établir comme nouvelle convention, en perdant toute énergie paradoxale : «Rien ne s'use plus vite que le sentiment de transgression » (Genette, cité par Schlickers, 2005, p. 166). À l'instar du cinéma, les périodes les plus propices au sentiment de transgressions métaleptiques pourraient être conditionnées par l'âge du média 
considéré : le temps de son émergence tout d'abord, où la grammaire narrative est en pleine adaptation par rapport à ce qui est connu par ailleurs, où les conventions de lecture ne sont pas établies et les effets de perception pas encore anticipés; puis bien après avec la phase de renouvèlement, avec un retour sur les codes établis, un travail d'auto référence et de réflexivité - travail appelé en introduction de ce texte « modernité ». ${ }^{8}$

\section{Du jeu vidéo réflexif au jeu sérieux}

\section{Figure d'exception pour le jeu vidéo}

19 En matière de réflexivité, sont à relever Deadpool - déjà cité pour ses références permanentes au fait d'être dans un jeu - et deux productions conçues par Davey Wreden : The Stanley Parable $(2013)^{9}$ et The Beginner's Guide $(2015)^{10}$. The Stanley Parable dont il sera essentiellement question ici est une puissante mise en abyme (Barnabé et Dozo, 2015) où le jeu est au cœur d'une critique croisée entre l'acte de développer un jeu et l'acte d'y jouer. Concrètement, un narrateur omniprésent commente chacun des choix du joueur, les lui reprochant parfois, à toutes fins de mettre en lumière la conception de jeu (game design), sa logique et ses écueils. Comme l'analyse l'équipe du Gamelab (2015), le jeu produit ainsi une critique globale du jeu vidéo, avec un accent particulier sur ce qui apparait parfois comme une trop grande linéarité. Concrètement, le joueur a vocation à déplacer un personnage à la $1^{\text {re }}$ personne dans des bureaux désertés - métalepse ontologique descendante purement fonctionnelle -, alors que le narrateur est essentiellement cantonné à raconter cette pérégrination, via une métalepse rhétorique ascendante qui a pour origine le niveau extradiégétique (N-1) et pour destination le joueur ( $\mathrm{N}$ 0). Pour le dire autrement, il y a deux types de métalepses à l'œuvre avec d'un côté interaction et de l'autre récit. Mais par ce récit précisément, le joueur comprendra que la liberté apparente de ses interactions est intégralement cadrée par l'environnement et les conditions organisées en amont par le concepteur. En résumé, l'histoire spatialisée préalablement écrite conduit l'interaction du joueur et parallèlement cette interaction déclenche le récit. Deadpool et les jeux de Davey Wreden font figure d'exception, en ce sens où la métalepse n'est ni seulement fonctionnelle, ni utilisée de manière ponctuelle, mais inscrite au cœur de la jouabilité (gameplay) pour articuler histoire, interaction et récit. Comme le dit Turcev (2014) pour Deadpool, ces exemples nous mettent enfin « face à la particularité interactive de la construction du récit dans le jeu vidéo». Cette inscription dans la jouabilité implique que la fréquence de la métalepse soit élevée, s'écartant là encore de l'effet de « briser le $4^{\mathrm{e}}$ mur », lequel n'existe que dans la rareté.

\section{Jeu sérieux et impératif réflexif}

Sans comparaison possible du point de vue de l'audience médiatique, la déclinaison utilitaire du jeu vidéo nommé jeu sérieux ou serious game se doit nécessairement d'apporter une réflexivité pour assurer sa visée d'apprentissage. De précédents travaux ont exploré le potentiel des métalepses, avec notamment des regards caméra déclenchés par l'interaction (Allain et Szilas, 2012), ainsi qu'une mise en abyme particulière qui lie par des métalepses choix du joueur et actes du personnage (Allain, 2013a, 2013b). EHPAD ${ }^{\mathrm{SG}}$ (Dæsign, 2012) ${ }^{11}$, l'un des jeux spécifiquement construits autour de ces principes met en scène un " avatar » d'un genre particulier : s'il ressemble au joueur (métier, fonctions) et partage les mêmes buts, il se distingue de l'avatar traditionnel en manifestant une 
autonomie de pensée. La position classique du joueur souhaitant " piloter » le personnage à la $3^{e}$ personne est déstabilisée lorsque celui-ci lui se retourne pour adresser des commentaires sur les choix entrepris. La transgression cherche ici à mettre en question les présupposés du joueur, à le faire progresser dans sa réflexion en provoquant une distanciation et des déstabilisations cognitives ciblées. Elle cherche aussi à renforcer les liens entre les mondes, autrement dit à mieux transférer les apprentissages dans le quotidien. En outre, le personnage annoncera qu'il porte la parole d'une équipe d'experts, hors du jeu, qui a réfléchi aux bonnes pratiques qu'il convient d'appliquer. Cette entreprise de déstabilisation et de rapprochement du réel validée expérimentalement pour le jeu sérieux (Allain, 2013) répond en partie au questionnement de Giner (2017) s'interrogeant sur la manière dont le jeu vidéo et ses formes "persuasives" ou " expressives" peuvent «susciter la réflexivité chez les joueurs et les joueuses et [...] l'orienter vers ce qu'ils vivent dans leur vie quotidienne » (p. 167). Aux finalités évoquées plus haut indiquant que la métalepse peut surprendre, déranger, inquiéter, ou amuser s'ajoute donc celle de conduire l'apprentissage.

\section{Réfléchissement de la transgression}

\section{De l'effet transitif à l'effet miroir}

Les jeux vidéo cités comme exceptions semblent au sommet de leur art, tant ils sont capables d'intégrer une réflexion et une mise en interaction de la nature du support jeux vidéo: la dimension fonctionnelle y est à la fois récupérée et discutée par le récit. Toutefois, deux limites sont à relever. La première est le côté systématique du procédé, dont l'énergie paradoxale s'affaiblit avec la répétition comme évoquée précédemment. Il semble alors que l'effet de transgression se maintienne moins à l'échelle du jeu qu'à l'échelle du domaine vidéoludique, ces productions affichant une singularité. La seconde limite est que ces exemples fonctionnent sur un mode que l'on peut qualifier de " classique »: dans la veine du $4^{\mathrm{e}}$ mur, la remise en question des structures canoniques vise encore à surprendre le joueur de manière frontale. L'effet recherché leur est directement et uniquement destiné.

En contrepoint, il est dès lors intéressant d'investir une autre perspective, en délaissant l'ambition d'un effet transitif et en explorant des récits interactifs dans lesquels la transgression serait d'abord perçue par les personnages eux-mêmes: si les métalepses fonctionnelles ne surprennent plus le joueur et si leur répétition épuise les autres, le récit interactif a par contre tout le loisir de simuler la transgression au sein du monde vidéoludique, avant que celui-ci n'agisse par mimétisme et empathie sur le joueur. De ce point de vue, les jeux vidéo évoqués jusque-là laissent le champ libre, presque complètement en friche ${ }^{12}$ : avec les jeux de Davey Wreden, la chose est entendue, car il n'y a aucun personnage pouvant assurer un relai diégétique; le joueur ne pourra déceler une transgression vécue depuis l'univers du jeu. Avec Deadpool, l'arrogance du personnage principal le place dans une position surplombante par rapport au joueur, mettant Deadpool à l'abri de toutes surprises; de plus les autres personnages ne semblent pas concernés par les choix directs du joueur. Il faut donc aller au-delà. 


\section{Propositions d'effets miroir} prophète explique au héros qu'il n'est qu'un pantin, piloté par des forces supérieures (quasi métalepse). C'est aussi partiellement le cas avec Assassin's Creed II (Ubisoft, 2009)13 lorsque que Minerva (PNJ) se détourne d'Ezio (PJ), pour s'adresser face caméra au joueur ; jusque-là, simple rupture classique apparentée au $4^{\mathrm{e}}$ mur, mais Ezio réagit ensuite avec surprise, regardant autour de lui et disant: «De qui [à qui] parlez-vous? Je ne vois personne d'autre ici... ». Si par hasard la métalepse rhétorique ascendante de Minerva ne surprenait pas directement le joueur, elle est redoublée par la surprise du PJ, et ainsi réfléchie auprès du joueur qui s'identifie au moins partiellement à lui. Le développement de ces perspectives non plus sur une séquence de fin de jeu comme ici, mais sur la longueur du jeu, est encore à rapporter. Les exemples cités ayant été répertoriés de manière incidente en explorant le principe du $4^{\mathrm{e}}$ mur, il est probable qu'une recherche orientée ouvrirait un vaste champ d'analyse. 
Si les premiers modèles présentés ci-dessus sont encore sommaires, ils ouvrent un nombre important de combinaisons en croisant les seuls critères distinctifs de position, orientation, direction et les différents niveaux ou espaces concernés. Et que dire si s'ajoutent la localisation dans la temporalité du récit, la fréquence et la durée ${ }^{{ }^{14}}$ Les modèles ont donc vocation à se multiplier et s'affiner ${ }^{15}$ Le travail à conduire est d'autant plus monumental qu'il aura à intégrer la sphère récemment ouverte par le transmédia, les projets en réalité alternée (ARG), les jeux en réalité virtuelle (VR) et ceux en réalité augmentée (AR). Pour l'heure et considérant le présent corpus, par ses qualités mimétiques et empathiques, par son interactivité, et par la puissance de la mise en abyme, le jeu vidéo est en capacité d'utiliser la métalepse pour que le joueur soit le destinataire second de transgressions qu'il a initiées. Cette approche fait écho au principe « d'expressivité » du jeu vidéo au sens où « l'expressivité provient à la fois des procédures induites par la structure du jeu et des actions menées par le joueur » (Genvo, 2013, p. 128). Ces propositions font aussi écho aux aspirations D'Argenio (2016a) espérant que « des studios AAA tenteront de faire un jeu où vous, le joueur, serez une partie importante de l'univers $d u$ jeu $»^{16}$. Plus largement, elles répondent à la critique qu'il esquissait en parlant d'un " $5^{\mathrm{e}}$ mur " séparant de plus en plus la dimension interactionnelle de la dimension narrative, expliquant que «nous ne jouons plus l'histoire de nos plus grands jeux [mais] qu'elle nous est racontée ». En l'occurrence, les modèles présentés ouvrent des perspectives à la fois de l'ordre du théorique et de la conception pour tresser ensemble ludique et narratif. Ils répondent à notre seconde hypothèse (H2) qui avançait que l'omniprésence de la métalepse dans le jeu vidéo lui impose de renouer avec la narration si elle veut offrir à nouveau un sentiment de transgression.

\section{Conclusion}

Si les métalepses sont consubstantielles au jeu vidéo, elles se cantonnent bien souvent à actionner le plan ludique pour assurer des transactions les plus transparentes possible entre le joueur et l'univers fictionnel. Pour celles qui se veulent plus transgressives, notre analyse a montré qu'elles se sont trouvées confondues sous l'expression "briser $4^{\mathrm{e}}$ mur ", laquelle regroupe au mieux des formes éculées pour solliciter la mémoire collective des communautés, ou regroupe au pire des simulacres vidés de sens. Occupant encore un large espace médiatique, cette expression souvent convoquée à tort a mis dans son ombre des transgressions bien plus novatrices et spécifiques au média. Or, la transgression d'un genre ou d'un domaine ne réclame pas nécessairement une forme spectaculaire : c'est son effet sur les destinataires qui prime et celui-ci peut prendre des chemins détournés, jusque dans les tréfonds des niveaux narratifs. De vastes champs de créations et d'analyses sont à poursuivre. Bien sûr, tous les jeux vidéo n'ont pas vocation à renouveler le domaine et tous ne cherchent pas à conduire une narration. Pour les autres toutefois, des formes alternatives de métalepses sauront renouveler le sentiment de transgression, pour le mettre au service de la jouabilité ou pour le mettre plus largement au service du jeu vidéo et ses lettres de noblesse. Dès lors, le joueur n'est plus forcément le destinataire premier de ces métalepses, mais il est à la fois à l'initiative du point de vue fonctionnel et destinataire second du point de vue du récit. Et si ce ne sont les jeux de loisir et de divertissement qui souhaiteront bénéficier de ces micro révolutions, misons que les jeux pour se former ou développer son esprit s'y attarderont. 


\section{BIBLIOGRAPHIE}

ALLAIN S. (2013a), « La mise en abyme actée, nouveau fer-de-lance du serious game », Revue d'Interaction Homme-Machine, 14-1, pp. 33-64, http://europia.org/RIHM/V14N1.htm

ALLAIN S. (2013b), Serious game et perception du réel : lecture documentarisante et potentiel cognitif. Thèse des Universités de Genève et Grenoble, http://archive-ouverte.unige.ch/unige:30964 ALLAIN S., et Szilas, N. (2012), «Exploration de la métalepse dans les "serious games" narratifs », Revue Sticef, 19., http://sticef.univ-lemans.fr/num/vol2012/07-allain/sticef_2012_allain_07.htm BARNABÉ F. et DOZO B.-O. (2015), « La mise en abyme vidéoludique comme vecteur d'une immersion au second degré : une analyse du jeu The Stanley Parable ", Colloque Sonder l'abyme. la mise en abyme dans les textes et les images, Université de Luxembourg, 16 et 17 janvier 2015, http:// orbi.ulg.ac.be/handle/2268/176816

BEAULIEU S. (2015), « Introduction au quatrième mur », Métafictions.fr, 24 juin, http:// metafictions.fr/introduction-au-quatrieme-mur/

BLANCHET A. (2008), «Cinéma et jeux vidéo : trente ans de liaisons », Médiamorphoses, 22, pp. 33-38.

BOUCHARDON S. (2009), Littérature numérique : le récit interactif. Paris, Hermès Science.

COHN D. (2005), « Métalepse et mise en abyme », in J. Pier. et J.-M. Schaeffer (dir.), Métalepse : entorses au pacte de la représentation, pp. 121-130, Paris, EHESS.

CONWAY S. (2009), A Circular Wall? Reformulating the Fourth Wall for Video Games, http:// www.gamasutra.com/view/feature/132475/a_circular_wall_reformulating_the_php?print=1

D'ARGENIO A. (2016a), The Inescapable Link between Game and Story, http:// www.escapistmagazine.com/articles/view/video-games/features/15324-The-Link-betweenGame-and-Story-Breaking-the-Fourth-Wall

D'ARGENIO A. (2016b), Top 10 games that break the 4th wall, https://www.gamecrate.com/top-10games-break-4th-wall/14792

DI CROSTA M. (2009), Entre cinéma et jeux vidéo l'interface-film : métanarration et interactivité. Bruxelles, De Boeck.

GAMELAB (collectif), (2015,), « The Stanley Parable », 6 janvier http://www.expressivegame.com/ fr/analyses/the-stanley-parable/

GAMINGBOLT (collectif) (2016), 15 Insane Fourth Wall Breaks That BLEW Gamers Minds, https:// www.youtube.com/watch ?v =HpKF1GAArp4

GENETTE G. (2004a), La métalepse. De la figure à la fiction (entretien conduit par John Pier), http:// www.vox-poetica.org/entretiens/genette.html

GENETTE G. (2004b), Métalepse : de la figure à la fiction. Paris, Seuil.

GENETTE G. (2007), Discours du récit. Paris, Seuil.

GENVO S. (2013), « Comprendre et développer le potentiel expressif, Understanding and developing the expressive potential », Hermès, La Revue, 62, pp. 127-133. 
GIANT BOMB (collectif) (2017), « Breaking the Fourth Wall », Gamingbolt.com, 22 février https:// www.giantbomb.com/breaking-the-fourth-wall/3015-138/

GINER E. (2017), « Inciter à la réflexivité par les mécanismes ludiques : une analyse comparée de The Witness, Undertale et The Beginner's Guide », Le Pardaillan, 2, pp. 167-181.

GRIFFITHS J. (2016), « 15 Most Amazing Fourth Wall Breaks In Video Games », Gamingbolt.com, 5 août, http://gamingbolt.com/15-most-amazing-fourth-wall-breaks-in-video-games

Harpold T. (2007), « Screw the Grue: Mediality, Metalepsis, Recapture », Game Studies, 7-1, http:// gamestudies.org/0701/articles/harpold

HUIZINGA J. ([1938]1988), Homo ludens. Paris, Gallimard.

KRONSTRÖM M. (2001), « Le regard à la caméra », Cadrage.net, http://archive.is/vlxz4

OWEN E. (2013), « 8 Fantastic Fourth Wall Breaks That Blew Gamers Minds », 11 juillet http:// whatculture.com/gaming/8-fantastic-fourth-wall-breaks-that-blew-gamers-minds

PIER J. (2005), « Métalepse et hiérarchies narratives », in J. Pier. et J.-M. Schaeffer (dir.), Métalepse : entorses au pacte de la représentation, pp. 247-262, Paris, EHESS.

RYAN M.-L. (2004), « Metaleptic machines », Semiotica, 150-1, pp. 439-469.

RYAN M.-L. (2005), « Logique culturelle de la métalepse, ou la métalepse dans tous ses états », in J. Pier. et J.-M. Schaeffer (dir.), Métalepse : entorses au pacte de la représentation, pp. 201-224, Paris, EHESS

SCHLICKERS S. (2005), «Inversions, transgressions, paradoxes et bizarreries. La métalepse dans les littératures espagnole et française », in J. Pier et J.-M. Schaeffer (dir.), Métalepse : entorses au pacte de la représentation, pp. 151-166, Paris, EHESS.

TURCEV N. (2014), « Le quatrième mur : s'extirper de la binarité » Merlanfrit, http:// merlanfrit.net/Le-quatrieme-mur-s-extirper-de-la

VERNET M. (1988), Figures de l'absence : de l'invisible au cinéma, Paris, Cahiers du cinéma.

WATCHMOJO.COM (2014), Top 10 Fourth Wall Breaks in Video Games, https://www.youtube.com/ watch $? \mathrm{v}=$ WocjtVabfho

YOUNG J. (2016), « 18 Times Videogames Broke the 4th Wall a Little Too Hard », Dorkly.com, 29 novembre, http://www.dorkly.com/post/81375/times-videogames-just-dngaf-about-the-4thwall

\section{NOTES}

1. Par convention, le terme "texte» englobe tout aussi bien un écrit, un film, une pièce de théâtre ou un jeu vidéo. De la même manière, le terme « lecteur » s'étend aussi aux spectateurs et aux joueurs.

2. https://www.mdcu-comics.fr/news-0020598-commencer-les-comics-commencer-les-comicsdeadpool.html

3. Le corpus restreint est disponible en ligne à l'adresse suivante: www.apt-a.com/uni/ metalepsis-of-video-game.html

4. L'ensemble des conclusions est disponible en ligne aux côtés du corpus.

5. La série Pokémon a donné lieu à un recensement impressionnant à l'adresse suivante : http:// bulbapedia.bulbagarden.net/wiki/Breaking_the_fourth_wall 
6. On mentionnera une contribution voisine avec Harpold (2007) et la « recapture » des éléments issus ou périphériques au jeu pour les intégrer au dispositif.

7. Ces mises en scène persisteront-elles encore avec des jeux utilisant des masques de réalité virtuelle?

8. Pistes dégagées lors de la journée d'étude sur la métalepse, organisée par Nicolas Szilas, en présence de Marie-Laure Ryan, Urs Richle et Sébastien Allain, le 6 avril 2011, à Genève.

9. Des descriptions, de nombreux extraits audio et une analyse sont disponibles à partir de la $38^{\mathrm{e}}$ minute dans le podcast Profil Ludique, S01E03, «Le walking simulator : Jeu ou non-jeu? » https:// soundcloud.com/profil-ludique/profil-ludique-s01e03-jeu-ou-non-jeu-telle-est-la-

question\#t=38:02

10. Voir les descriptions et l'analyse de The Beginner's Guide par Giner (2017).

11. Voir la comparaison entre la version classique et la version collaborative à partir de 1 minute 20 secondes http://dai.ly/xqmozt

12. À l'évidence puisqu'ils visent une métalepse ascendante et non descendante.

13. Séquence disponible à cette adresse https://youtu.be/jpJ0lvC_ka4?t=2m12s

14. La rencontre à l'École Normale Supérieure entre Mathieu Triclot et Martin Lefebvre en janvier 2016 a listé une série de jeux réflexifs qu'il serait intéressant de qualifier suivant ces critères. Voir vidéo en ligne intitulée Méta-jeu - Quand le jeu vidéo réfléchit sur lui-même, https:// www.youtube.com/watch?v=GOhEMNzdi2o

15. Ce constat préfigure l'ouverture d'une base de données participative, communautaire et internationale pour assurer la classification des exemples venus aussi bien du monde amateur, que du monde du game design ou du monde académique. Les personnes souhaitant s'associer au projet peuvent se manifester à l'adresse suivante: http://www.apt-a.com/uni/metalepsisdb.html

16. Le lecteur appréciera ici la métalepse rhétorique employée par D'Argenio pour "vous» interpeller.

\section{RÉSUMÉS}

Parmi les transgressions narratives, la métalepse s'est déployée sur tous les médias, jusqu'à occuper une place prépondérante dans les récits vidéoludiques. Désormais, son omniprésence interroge la possibilité qu'elle produise encore un quelconque sentiment de transgression. Seraitelle à reléguer dans les usages conventionnels du jeu vidéo ? Cette contribution s'appuiera sur plusieurs disciplines (études littéraires, cinématographiques, narratologiques, ludologiques, communicationnelles et didactiques) pour mettre de côté les métalepses purement fonctionnelles du jeu vidéo et s'attacher à différentes formes articulant ludique et narratif. Après avoir présenté le concept de métalepse dans une première partie, la seconde partie s'intéressera à une transgression dite $\mathrm{du}$ " $4^{\mathrm{e}}$ mur ». Expression fréquemment utilisée, il s'avèrera qu'elle est non seulement héritée des médias antérieurs - et donc peu spécifique au jeu vidéo -, mais surtout qu'elle masque une diversité de procédés. Sur la base d'un corpus d'une vingtaine de jeux, la dernière partie valorisera des métalepses trop peu mises en lumière, si ce n'est impensées. En dépit de leur faible popularité actuelle, celles-ci pourraient bien renouveler le sentiment de transgression d'antan, tout en participant à la modernité du média jeu vidéo. 
Among the narrative transgressions, the metalepsis has spread over all the media, until occupy a prominent place in video games. Henceforth, its omnipresence questions about the possibility that it still produces any sense of transgression. Would it be relegated to the conventional uses of video games? Based on several disciplines (literary, cinematography, narratology, game studies, communication and didactic studies), this contribution will put aside the purely functional metalepsis of video games to focus on different forms which articulate playful and narrative. After presenting the concept of metalepsis in the first part, the second one will discuss a transgression known as the "fourth wall". It will be shown that this frequently used expression is not only inherited from previous media - and therefore not specific to video games - but that it masks above all a variety of processes. From a corpus of twenty games, the last part will focus on some metalepsis not well-known, if not thought out. In spite of their current low popularity, these metalepsis could well renew the feeling of transgression while participating in the modernity of the video games.

\section{INDEX}

Mots-clés : métalepse, transgression, narratologie, quatrième mur, jeux sérieux, modernité, expressivité, empathie

Keywords : metalepsis, transgression, narratology, fourth wall, serious games, modernity, expressiveness, empathy

\section{AUTEUR}

\section{SÉBASTIEN ALLAIN}

Université Savoie Mont-Blanc, laboratoire LLSETI 